\title{
Age-Related Macular Degeneration and Cardiovascular Diseases and Risk Factors Associated with It - A Cross Sectional Study in Kerala
}

\author{
Deepa M.G. ${ }^{1}$, Sija S. ${ }^{2}$, Jacob K. K. ${ }^{3}$, Rajeevan P. ${ }^{4}$ \\ ${ }^{1}$ Department of Ophthalmology, Government Medical College, Ernakulam, Kerala, India. ${ }^{2}$ Department of \\ Ophthalmology, Government Medical College, Alleppey, Kerala, India. ${ }^{3,4}$ Department of Ophthalmology, \\ Government Medical College, Kottayam, Kerala, India.
}

\section{ABSTRACT}

\section{BACKGROUND}

Age related macular degeneration [AMD / ARMD] is the leading cause of irreversible blindness in the developed world and accounts for $8.7 \%$ of all blindness globally. Despite being the most common cause of blindness worldwide, the importance of AMD in the poorest and most populous regions of Asia and Africa pales in comparison to other eye diseases like cataract, glaucoma and childhood blindness. AMD has been excluded from the list of priority eye diseases as defined by VISION 2020. The right to sight, bears testament to the current situation. Moreover, there is no early administered or inexpensive therapeutic approach to AMD. Hence it will doubtless be a major contributor to visual impairment in the elderly population in the years to come. This highlights the need to research for factors which if modified might alter the prognosis and outcome. The probability of AMD being a marker to predict associated systemic morbidity augments the need for extensive studies.

\section{METHODS}

A cross sectional study was done at a tertiary care center in Kerala from May 1, 2009 to December 31, 2010. The study included all patients attending the ophthalmology OPD at the hospital who were found to have clinical features of AMD / ARM during the study period. The data was analyzed with SPSS.

\section{RESULTS}

The percentage of coronary artery disease [CAD] in our study was $8.18 \%$ as opposed to $7.5 \%$ in rural and $12 \%$ in urban population of Kerala and hence showed no increased occurrence in AMD patients. Of the various risk factors analyzed, type 2 diabetes mellitus had an increased percentage of occurrence in AMD patients. So, the disease may be associated with AMD but association between them is not statistically significant $(\mathrm{p}=$ 0.2586). Smoking, alcohol intake, hypertension, hypercholesterolemia had comparable prevalence in AMD and general population. Meanwhile, the dietary habits analyzed revealed that taking a predominant vegetarian diet did not offer any significant protective effect.

\section{CONCLUSIONS}

AMD is considered to have a multifactorial aetiology. Since none of the risk factors analyzed could find a statistically significant correlation to AMD or its severity, the relevance of these factors needs further investigation.

\section{KEY WORDS}

Age Related Macular Degeneration, Coronary Artery Disease, Type 2 Diabetes Mellitus
Corresponding Author:

Dr. Sija $S$,

Pranamam, CSM Nagar,

H. No. 99, Edapazhanji - 695010,

Thiruvananthapuram, Kerala,

India.

E-mail:drsija1981@gmail.com

DOI: $10.14260 / \mathrm{jemds} / 2020 / 663$

How to Cite This Article:

Deepa MG, Sija S, Jacob KK, et al. Agerelated macular degeneration and cardiovascular diseases and risk factors associated with it - a cross sectional study in Kerala. J Evolution Med Dent Sci 2020;9(41):3024-3028, DOI: $10.14260 / \mathrm{jemds} / 2020 / 663$

Submission 28-06-2020,

Peer Review 03-09-2020,

Acceptance 09-09-2020,

Published 12-10-2020.

Copyright $(2020$ Deepa M. G. et al. This is an open access article distributed under Creative Commons Attribution License [Attribution 4.0 International (CC BY 4.0)] 


\section{BACKGROUND}

Age related macular degeneration is the leading cause of irreversible blindness in the developed world and accounts for $8.7 \%$ of all cases of blindness globally.1,2 The overall prevalence is projected to increase by more than $50 \%$ by the year 2020.

Despite being the third most common cause of blindness worldwide the importance of AMD in poorest and the most populous regions of Asia \& Africa pales in comparison with the impact of other eye diseases like cataract, glaucoma and childhood blindness. The exclusion of AMD from the list of priority eye diseases as defined by VISION 2020: The right to sight bears testament to the current situation.

AMD can be defined as degenerative changes at the macula characterised by the presence of the following:

- Drusen.

- RPE abnormalities like hypopigmentation / hyperpigmentation associated with drusen.

- $\quad$ RPE and associated neurosensory detachment.

- Geographic atrophy ie sharply delineated confluent areas of hypo or hyperpigmentation or apparent absence of RPE in which choroidal blood vessels are more visible than surrounding areas. , $^{3,4}$

- Choroidal neovascularisation with or without subretinal haemorrhages or exudation.

\section{Classification of AMD}

- Early AMD: presence of $<20$ medium size drusen or RPE anomalies.

- Intermediate AMD: at least one large drusen or numerous medium sized drusen or geographic atrophy that doesn't extend to the centre of macula.

- $\quad$ Advanced / late AMD: choroidal neovascularisation and its sequelae like haemorrhages / exudation / scarring.5,6,7

The risk factors include advancing age, race [more in whites] ${ }^{11}$ genetic factors like polymorphism in complement factor $\mathrm{H}[\mathrm{CFH}]$ gene located on chromosome 1 [1q 31] which increases F29 risk, 8,9,10,11,12 smoking, obesity, ${ }^{13,14}$ low intake of green leafy vegetables, vitamin $\mathrm{A}$, high intake of fish and nuts, ${ }^{15,16}$ hypertension, CAD, hypercholesterolemia, diabetes mellitus].

The main hypothesis about aetiology include

- Antioxidant Hypothesis - Oxygen free radicals cause lipid peroxidation which damage photoreceptors which end up in lipofuscin accumulation and later on drusen. ${ }^{17}$

- Atherosclerotic Hypothesis - Atherosclerotic changes in sclera and Bruch's membrane can affect chorio capillary circulation there by increasing AMD.18

- $\quad$ Photo Toxicity Hypothesis - Blue light increases oxidation and hence damage the macula.

Management strategies include Antioxidant supplementation according to AREDS 2 study, lifestyle and dietary modifications [like decreased fat intake, high intake of beta carotene, vitamin C,E, Zinc, fish and long chain PUFA, quitting smoking etc.], photo dynamic therapy [PDT ${ }^{19}$ ], Trans pupillary thermotherapy [TTT], Intra vitreal anti angiogenic therapy, ${ }^{20}$ Intravitreal steroids ${ }^{21}$ and vitreo retinal surgeries like sub macular procedures, macular translocation, ${ }^{22}$ pneumatic displacement of sub macular haemorrhage etc. The evolving approaches include combination therapies using multiple methods mentioned above, genetic approaches like intra vitreal gene transfer, implanting intraocular device ${ }^{23}$ to improve vision or may retrieve functional vision etc.

Moreover, at present there is no easily administered and inexpensive therapeutic approach to AMD. Hence, it is doubtless to be a major contributor of visual impairment in the elderly population in the years to come. This scenario has led to a search of factors which if modified might alter the prognosis. Many factors have been incriminated suggesting a multifactorial aetiology for the disease.

I sincerely hope that this study will highlight the importance of proper screening for AMD which could reduce the visual and associated systemic morbidity by timely intervention and lifestyle modifications.

\section{Objectives}

1. To study the demographic factors like age and sex in AMD.

2. To assess if there is any association of AMD with cardiovascular diseases (CAD) and risk factors like hypertension, diabetes mellitus, obesity and overweight, hyperlipidaemia, tobacco use, alcohol use and dietary habits.

\section{METHODS}

This is a cross sectional study, conducted at Medical College Hospital, Kottayam, during the time period May 1, 2009 December 31, 2010. All patients attending ophthalmology OPD Medical College, Kottayam and found to have clinical features of age-related maculopathy and AMD during the study period. The diagnostic criteria for AMD were clinical features suggestive of early AMD like, 20 medium sized drusen at macula, RPE pigmentary abnormalities and late AMD like geographic atrophy, PED, CNV and its sequelae like haemorrhage, exudation / scarring on fundus examination by direct ophthalmoscope / indirect ophthalmoscope / slit lamp bio microscopy with 90D / 78D lens. A documented history or a positive result in Echocardiography or tread mill test [TMT] whichever is indicated in the patient and diagnosed by a cardiologist was considered positive as CAD.

\section{Exclusion Criteria}

Patients with diabetic retinopathy and other macular diseases like toxic or solar maculopathy.

\section{Data Collection}

A thorough history with reference to the factors to be evaluated were collected with a pro forma including age, sex, previous illness, history of hypertension, diabetes mellitus, coronary artery disease, drug intake, dietary habits including 
whether vegetarian or non-vegetarian and among nonvegetarians whether they took predominantly meat / fish / vegetables including green leafy vegetables (use $>3$ days in a week and $>2$ servings / day), and habit of smoking or alcohol intake.

Examination of pulse, BP, BMI, Systemic examination of CNS, CVS, GIT, Respiratory systems was done. Ocular examination included direct and indirect ophthalmoscopy, slit lamp bio microscopic examination and evaluation with fundus photo, fundus fluorescein angiography and indocyanine green if indicated. Investigations included RBS, ECG, Fasting lipid profile, echocardiography or TMT (Tread Mill Test).

\section{Statistical Analysis}

Data entered into Microsoft Excel work sheet and analysis done with CDC Epi info. Univariate analysis done using chisquare test. The proportion of patients was found as frequency percentage. Odds ratio was used for risk factors shown to be significant on univariate analysis. A probability [p] of less than 0.05 was considered to be significant.

\section{RESULTS}

A total of 110 patients was studied of which 48 were males and 62 were females.

\begin{tabular}{|ccc|}
\hline Age Group & Frequency & Percent \\
$40-50$ & 6 & $5.5 \%$ \\
$50-60$ & 19 & $17.3 \%$ \\
$60-70$ & 44 & $40.0 \%$ \\
$>70$ & 41 & $37.3 \%$ \\
Total & 110 & $\mathbf{1 0 0 . 0} \%$ \\
\hline Table 1. Age Wise Distribution of Patients with AMD \\
\hline
\end{tabular}

The proportion of CAD in our study was 8.18 percentage [ 9 out of 110 patients studied] Advanced AMD was more in CAD patients than in non-CAD patients but not statistically significant with [ $p-0.2221]$. Among the various risk factors analysed, diabetes mellitus had a higher prevalence in AMD patients. Smoking, alcohol intake, hypertension and hypercholesterolemia had comparable prevalence in AMD and general population. Contrary to many other studies, taking a predominantly vegetarian diet did not offer any significant protective effect.

\section{Distribution of CAD in AMD Patients}

Among 110 patients studied 9 had CAD i.e. $8.18 \%$. The prevalence of CAD in general population in India is $4-11 \%$ and in South India it is $6-7.4 \%$. According to the registry under cardio logical society of India in Kerala the prevalence is $7.5 \%$ in rural and $12 \%$ in urban population. ${ }^{24,25}$ Advanced AMD had more prevalence (44.4\%) in CAD than among the non-CAD patients (26.7\%) but it is not statically significant as the $\mathrm{p}-0.2221(>0.05)$. In Singapore Eye Study it was found that $29 \%$ of patients with early AMD developed CAD over a 7year period as compared to $18 \%$ in normal individuals. The mechanism proposed was due to common risk factors and pathogenesis of chronic inflammation and endothelial dysfunction.
An association between CAD and advanced AMD was cited in a study in France in the National Health and Nutrition examination survey, Rotterdam study. Our study showed prevalence of CAD in AMD patients comparable to that of normal population. Though not statistically significant increased prevalence of advanced AMD was noted in CAD patients than in normal group. In Indian scenario though the Andhra Pradesh Eye Disease Study \& Aravind Comprehensive Eye Disease Study showed an association between smoking and AMD, it was not statically significant. ${ }^{26}$

\begin{tabular}{|cccc|}
\hline CAD & Adv. AMD & Early AMD & Total \\
CAD & 4 & 5 & 9 \\
Row \% & 44.4 & 55.6 & 100.0 \\
No CAD & 27 & 74 & 101 \\
Row \% & 26.7 & 73.3 & 100.0 \\
TOTAL & $\mathbf{3 1}$ & $\mathbf{7 9}$ & $\mathbf{1 1 0}$ \\
Row \% & $\mathbf{2 8 . 2}$ & $\mathbf{7 1 . 8}$ & $\mathbf{1 0 0 . 0}$ \\
\hline \multicolumn{4}{c|}{ Early / Adv. } \\
\hline \multicolumn{4}{c}{ Table 2. Distribution of CAD in AMD Patients } \\
\hline
\end{tabular}

Distribution of AMD According to Predominant Diet Habit Majority of patients in the study were non vegetarians (71.8 $\%)$. Both early and advanced AMD showed increased prevalence among non-vegetarians. But on detailed analysis of diet pattern among non-vegetarians the prevalence was found in advanced (35.5\%) and early AMD (30.4). Also, there is no statically significant correlation between severity of AMD and diet pattern ( $p$ - 0.8692). This contradicts the hypothesis that diet rich in vegetables especially green leafy vegetables have a protective role in AMD.

\begin{tabular}{|cccc|}
\hline Early / Adv. & $\begin{array}{c}\text { Non-Vegetarian Diet } \\
\text { with Predominantly } \\
\text { Vegetables }\end{array}$ & $\begin{array}{c}\text { Non-Vegetarian Diet } \\
\text { with More Meat } \\
\text { Consumption }\end{array}$ & $\begin{array}{c}\text { Non-Vegetarian } \\
\text { Diet with More Fish } \\
\text { Consumption }\end{array}$ \\
Adv AMD & 9 & 4 & 7 \\
Row \% & 29.0 & 12.9 & 22.6 \\
Early AMD & 22 & 9 & 24 \\
Row \% & 27.8 & 11.4 & 30.4 \\
TOTAL & 31 & 13 & 31 \\
Row \% & 28.2 & 11.8 & 28.2 \\
\hline & & M / F / V & \\
\hline
\end{tabular}

Table 3. Distribution of AMD According to Predominant Diet Habits

Several studies have shown that a low intake of fruits and vegetables rich in Vitamin A increase the risk for AMD and that increased ? carotenes reduced AMD by reducing the photic damage by blue light as they form yellow pigment.

\section{Distribution of Diabetes Mellitus and AMD}

Out of 110 AMD patients studied 29 had diabetes mellitus (26.4\%). General prevalence of diabetes in India is $14 \%$ and in Kerala 11 - $16 \%$. This suggests an increased prevalence of diabetes in AMD patients. In advanced AMD group $32.3 \%$ were diabetic as compared to $24.1 \%$ in early AMD. So, the disease may be associated with AMD but the association between diabetes and severity of AMD is not statistically significant ( $p-0.2586)$.

\begin{tabular}{|cccc|}
\hline Early / Adv & Diabetic & Non Diabetic & Total \\
Adv AMD & 10 & 21 & 31 \\
Row \% & 32.3 & 67.7 & 100.0 \\
Early AMD & 19 & 60 & 79 \\
Row \% & 24.1 & 75.9 & 100.0 \\
TOTAL & 29 & 81 & 110 \\
Row \% & 26.4 & 73.6 & 100.0 \\
\hline \multicolumn{4}{|c}{ Table 4. Distribution of Diabetes Mellitus and AMD } \\
\hline
\end{tabular}




\section{DISCUSSION}

A total of 110 patients was studied of which 48 were males and 62 were females. The maximum numbers of AMD patients were in the age group of $60-70$ in this study. According to a study on CAD and its risk factors in south India prevalence of CAD was also maximum in the age group $55-70$ years with a p - 0.02 and hence significant.

Out of 110 only 7 patients had BMI > 25 [6.3\%] which is much less compared to prevalence of obesity in general population [45\%\} Majority of the patients had early AMD whereas increased percentage of advanced AMD was in age group $60-70$ yrs. (38.6) than in $>75$ yr. (24.4) contrary to many previous studies showing increased prevalence of late AMD in age $>75$ yrs. According to the INDEYE study, the prevalence of late AMD in $40-70$ years age group is $1.2 \%$ and in age group of $>75$ it is $2.5 \%$. It also proposed an increasing prevalence of advanced AMD with increasing age.

8.18 percent patients had CAD, comparable to the general prevalence of $4-11 \%$ in India and $6-7.4 \%$ in south India. According to the registry under cardio logical society of India, the prevalence in Kerala is $7.5 \%$ in rural and $12 \%$ in urban population. Advanced AMD had more prevalence in CAD [44.4 $\%$ ] than in non-CAD patients [26.7 \%] but not statistically significant p - 02221]. In Singapore Eye Study it was found that $29 \%$ of patients with early AMD developed CAD over a 7-year period as compared to $18 \%$ in normal individuals. The mechanism proposed was due to common risk factors and pathogenesis of chronic inflammation and endothelial dysfunction. An association between CAD and advanced AMD was cited in a study in France in the National Health and Nutrition examination Survey, Rotterdam Study. In our study, though not statistically significant increased prevalence of advanced AMD was noted in CAD patients than in normal group.

Though $23.6 \%$ of AMD patients were smokers, there doesn't seem to be an association between smoking and severity of the disease [ $p$ - 0.458]. Many previous Indian studies also found the same. A study at SCTIMS, Trivandrum has estimated the prevalence in Kerala to be $29 \%$. New Zealand study found an association of smoking with AMD as $26.8 \%$.

Majority of patients were non vegetarian (71.8\%) but association between the severity of disease and nonvegetarian diet is not statistically significant [p - 0.538]. A detailed analysis on diet pattern among non-vegetarians showed a higher prevalence among those who took predominantly vegetables [ $35.5 \%$ and $30.4 \%$ in advanced and early AMD). This contradicts the hypothesis that diet rich in vegetables especially green leafy vegetables have a protective role in AMD. Several studies have shown that low intake of fruits and vegetables rich in Vitamin A increase the risk of AMD and increased beta carotenes reduce AMD by reducing the photic damage by blue light as they form yellow pigment. According to Blue Mountain Eye Study, taking 1 serving / day /per week of fish reduce the risk of AMD [RR - $0.6995 \% \mathrm{CI}$ $0.49-0.98$ ]. Consumption of 1 serving per day of dark GLV decrease risk of AMD as per Diana Tang Paul Mithchell et al.

Out of 110 AMD patients (26.4\%) had diabetes mellitus whereas the general prevalence was only $16 \%$ in Kerala. Diabetes related changes in the function and structure of RPE, Bruchs membrane and choroidal circulation have been hypothesised to increase the risk of AMD. Histopathological studies in the eyes of diabetics have shown thickening of the basement membrane of choriocapillary walls and luminal narrowing and drop out of choriocapillaris and thickening of Bruchs membrane attributed to hyperglycaemia. In a systematic review and meta-analysis by Xue Chen etal. PosOne. 2014, many studies showed DM as a risk factor for AMD. [OR - 1.05,95 / 5CI, 1.00 - 1.14], stronger for late AMD than early AMD. Jae Kyung Choi et al study has found out significant correlation between DM and AMD in Korean population. OR r1.87CI - $95 \% 1.07$ - 3.28]. The EUREYE study too showed positive association between DM and Advanced AMD. But the association between disease severity and diabetes was not statistically significant. According to the Barbados eye study, after adjusting for age there was a 2.7-fold increase in association between diabetes mellitus and late AMD. The Blue mountain Eye study also reported significant association. ${ }^{27}$

The occurrence of hypertension and dyslipidaemia was comparable to general prevalence. In Indian studies, according to APEDS and ACES, though an association was found between hypertension and AMD it was not statistically significant. While the Baevardam study showed a 3-times rise of AMD in hypertensive. Atherosclerosis might be hypothesized to increase AMD risk by decreasing the blood flow through choroidal vasculature and lipid deposition in Bruch's with a reduction of micro permeability leading to up gradation of VEGF. Inflammation is also postulated as a cause, according to Klein R Ding Y Klein BE et al in the Women's Health Initiative Study.

\section{CONCLUSIONS}

The study population was evaluated for CAD and its risk factors. The prevalence of $\mathrm{CAD}$ and these factors were identified. This was compared to the prevalence of these factors in the general population in our part of the country with that of various other studies. Prevalence of CAD in our study is $8.18 \%$ and that in the general population of Kerala is $7.5 \%$ in rural and $12 \%$ in urban population. So, there is no increased prevalence of CAD in AMD patients.

Of the various factors studied, smoking, alcohol intake, hypertension, and hypercholesterolemia have comparable prevalence in AMD patients and in general population. Diabetes mellitus has higher prevalence in AMD patients. Meanwhile dietary habits studied revealed that taking a predominantly vegetarian diet didn't offer any significant protective effect. None of the factors studied had statistically significant correlation with the severity of AMD. A definite association between the factors studied and occurrence of AMD could not be determined as there was no control group (without AMD) included in the study due to the feasibility issues and ethical issues of doing all the costly investigations like TMT, Echo, FFA / ICG. Hence, a cross sectional study including a control group in the future may yield significant results which can improve our disease understanding and patient care.

Financial or Other Competing Interests: None. 


\section{REFERENCES}

[1] Pascolini D, Mariotti SP, Pokharel GP, et al. 2002 Global update of available data on visual impairment: a compilation of population - based prevalence studies. Ophthalmic Epidemiol 2004;11(2):67-115.

[2] Congdon N, O'Colmain B, Klaver CCW, et al. Causes and prevalence of visual impairment among in the United States. Arch Ophthalmol 2004;122(4):477-85.

[3] Friedman DS, O'Colmain BJ, Munoz B, et al. Prevalence of age - related macular degeneration in the United States. Arch Ophthalmol 2004;122(4):564-72.

[4] Alfaro DV, Liggett PE, Mieler WF, et al. Age related macular degeneration: a comprehensive text book. Philadelphia: Lippincott Williams \& Wilkins 2006.

[5] Bird AC, Bressler NM, Bressler SB, et al. An international classification and grading system for age related maculopathy and age related macular degeneration: the international ARM Epidemiological study Group. Surv Ophthalmol 1995;39(5):367-74.

[6] De Jong PTVM. Age related macular degeration. N Engl J Med 2006;355(14):1474-85.

[7] Hageman GS, Luthert PJ, Chong VNH, et al. An integrated hypothesis that considers drusen as biomarkers of immune mediated process at the RPE - Bruch's membrane interface in agin and age - related macular degeneration. Prog Retin Eye Res 2001;20(6):705-32.

[8] Zarbin MA. Current concepts in the pathogenesis of age related macular degeneration. Arch Ophthalmol 2004;122(4):598-614.

[9] Donoso LA, Kim D, Frost A, et al. The role of inflammation in the pathogenesis of age related macular degenartion. Surv Ophthalmol 2006;51(2):137-52.

[10] Anderson DH, Mullins RF, Hageman GS, et al. A role for local inflammation in the formation of drusen in the aging eye. Am J Ophthalmol 2002;134(3):411-31.

[11] Bressler SB, Munoz B, Solomon SD, et al. Racial differences in the prevalence of age related macular degeneration: the Salisbury Eye Evaluation (SEE) Project. Arch Ophthalmol 2008;126(2):241-5.

[12] Hageman GS, Anderson DH, Johnson LV, et al. A common haplotype in the complement regulatory gene factor $\mathrm{H}$ (HF1/CHF) predisposes individuals to age related macular degeneration. Proc Natl Acad Sci USA 2005;102(20):7227-32.

[13] Khan JC, Thurlby DA, Shahid H, et al. Smoking and age related macular degeneration: the number of pack years of cigarette smoking is a major determinant of risk of both geographic atrophy and choroidal neovascularisation. $\mathrm{Br}$ J Ophthalmon 2006;90(1):75-80.

[14] Klein BE, Klein R, Lee KE, et al. Measures of obesity and age-related eye diseases. Ophthalmic Epidemiol 2001;8(4):251-62.
[15] Schaumberg DA, Christen WG, Hankinson SE, et al. Body mass index and the incidence of visually significant agerelated maculopathy in men. Arch Ophthalmol 2001;119(9):1259-65.

[16] Seddon JM, Cote J, Rosner B. Progression of age-related macular degeneration association with dietary fat, transunsaturated fat, nuts and fish intake. Arch Ophthalmol 2003;121(12):1728-37. [Erratum, Arch Ophthalmol 2004;122(3):426].

[17] Van Leeuwen R, Boekhoorn S, Vingerling JR, et al. Dietary intake of antioxidants and risk of age related macular degeneration. JAMA 2005;294(24):3101-7.

[18] SanGiovanni JP, Chew EY, Clemons TE, et al. The relationship of dietary lipid intake and age related macular degeneration in a case control study: AREDS report no. 20. Arch Ophthalmol 2007;125(5):671-9.

[19] Treatment of AMD with Photodynamic therapy [TAP] study group. Photodynamic therapy of subfoveal choroidal neovascularisation in AMD with verteporfin: one year results of 2 RCTs-TAP report. Arch Ophthalmol 1999;117(10):1329-45.

[20] Jager RD, Aiello LP, Patel SC, et al. Risks of intravitreous injection: a comprehensive review. Retina 2004;24(5):676-98.

[21] Algvere PV, Steen B, Seregard S, et al. A prospective study on intravitreal bevacizumab (Avastin) for neovascular age related macular degeneration of different durations. Acta Ophthal Scand 2008;86(5):482-9.

[22] Mruthyunjaya P, Stinnet SS, Toth CA. Change in visual function after macular translocation with 360 degrees retinotomy for neovascular age related macular degeneration. Ophthalmology 2004;111:1715-24.

[23] Hudson HL, Lane SS, Heie JS, et al. Implantable miniature telescope for the treatment of visual acuity loss resulting from end-stage age-related macular degeneration: 1 year results. Ophthalmology 2006;113(11):1987-2001.

[24] Gupta R, Prakash H, Gupta VP, et al. Prevalence of determination of coronary heart disease in rural population in India. Journal of Clinical Epidemiology 1997;50(2):203-9.

[25] Kutty VR, Balakrishnan KG, Jayasree AK, et al. Prevalence of coronary heart diaease in the rural population of Thiruvananthapuram district, Kerala, India. Int J Cardiol 1993;39(1):59-70.

[26] Krishnaiah S, Das T, Niramalan PK, et al. Risk factors for age-related macular degeneration: findings from the Andhara Pradesh Eye Disease Study in South India. Invest Ophthol Vis Sci 2005;46(12):9.

[27] Mitchell P, Wang JJ. Diabetes, fasting blood sugar and age related maculopathy: the Blue Mountain Eye Study. Aust NZ J Ophthalmol 1999;27(3-4):197-9. 